\begin{tabular}{|c|l|}
\hline Title & Phase unwrapping for noisy phase maps using rotational compensator with virtual singular points \\
\hline Author(s) & Tomioka, Satoshi; Heshmat, Samia; Miyamoto, Naoki; Nishiyama, Shusuke \\
\hline Citation & $\begin{array}{l}\text { A pplied Optics, 49(25), 4735-4745 } \\
\text { https://doi.org/A0.1364/A 0.49.004735 }\end{array}$ \\
\hline Issue Date & 2010 \\
\hline Doc URL & http://hdl.handle.net/2115/44812 \\
\hline Rights & ○ 2010 Optical Society of A merica, Inc. \\
\hline Type & article \\
\hline File Information & 619F7E47-C4F6-7368-A A BE976E026419A D_205317.pdf \\
\hline
\end{tabular}

Instructions for use 


\title{
Phase unwrapping for noisy phase maps using rotational compensator with virtual singular points
}

\author{
Satoshi Tomioka, ${ }^{1, *}$ Samia Heshmat, ${ }^{2}$ Naoki Miyamoto, ${ }^{3}$ and Shusuke Nishiyama ${ }^{1}$ \\ ${ }^{1}$ Faculty of Engineering, Hokkaido University, Kita 13 Nishi 8, Kita-ku, Sapporo, Hokkaido 060-8628, Japan \\ ${ }^{2}$ Graduate School of Engineering, Hokkaido University, Kita 13 Nishi 8, Kita-ku, Sapporo, Hokkaido 060-8628, Japan \\ ${ }^{3}$ Graduate School of Medicine, Hokkaido University, Kita 15 Nishi 7, Kita-ku, Sapporo, Hokkaido 060-8638, Japan \\ ${ }^{*}$ Corresponding author: tom @qe.eng.hokudai.ac.jp
}

Received 17 March 2010; revised 20 July 2010; accepted 23 July 2010; posted 23 July 2010 (Doc. ID 125410); published 26 August 2010

\begin{abstract}
In the process of phase unwrapping for an image obtained by an interferometer or in-line holography, noisy image data may pose difficulties. Traditional phase unwrapping algorithms used to estimate a two-dimensional phase distribution include much estimation error, due to the effect of singular points. This paper introduces an accurate phase-unwrapping algorithm based on three techniques: a rotational compensator, unconstrained singular point positioning, and virtual singular points. The new algorithm can confine the effect of singularities to the local region around each singular point. The phaseunwrapped result demonstrates that accuracy is improved, compared with past methods based on the least-squares approach. (c) 2010 Optical Society of America
\end{abstract}

OCIS codes: $\quad 030.4280,090.2880,100.3175,100.5088,110.5086,120.5050$.

\section{Introduction}

Interferometers or in-line holography techniques are widely applied for the purpose of quantitative measurement of two-dimensional optical distance distribution. In these systems, fringe patterns are measured by digital devices such as CCD cameras, and then phase information is retrieved by Fourier domain filtering [1-3] or phase shift techniques $[2, \underline{4}, 5]$. The retrieved phase map is a distribution of principal values of phase, derived from the natural logarithm of a complex-valued function representing a wavefront. This map is wrapped by the function into the bounded range, such as $(-\pi, \pi]$ radians. Because the optical distance is actually a continuous function and its range is not bounded, the retrieved, wrapped map must finally be unwrapped to estimate optical distance distribution, by applying phaseunwrapping algorithms. Many algorithms have been

0003-6935/10/254735-11\$15.00/0

(C) 2010 Optical Society of America proposed for phase unwrapping. Some of them are summarized in [6]. They are all based on the assumption that no phase difference between adjoining pixels exceeds a half cycle ( $\pi$ radians). When an original phase to be measured satisfies the sampling theorem, unwrapped results are satisfactory regardless of the integration path. In actual experimental data, however, some phase differences that do not satisfy the criterion typically are included. Such circumstances are the result of noisy data where a lot of speckle noise is observed, as well as that of real optical gaps in an object to be measured. The proposed phase-unwrapping algorithms are targeting to remove or to reduce the inconsistencies that cause variations in phase-unwrapping results, depending on the choice of unwrapping path. When there is a low signal-to-noise ratio (SNR) in a fringe pattern, represented as a positive, real-valued function, the retrieved wavefront represented by a complex-valued function has a correspondingly low SNR. In this case, the wrapped phase as a principal value has a large 
error, of maximum $\pi$ radians. The relation of error between the complex function and the wrapped phase is described in [7].

The phase-unwrapping algorithms are classified into two types, according to the nature of the unwrapped result. The first is a reproducible type of algorithm, in which the rewrapped phase of the unwrapped result is identical to the original wrapped phase map, such as for path-following methods [8-14]. In these algorithms, the unwrapping operation is carried out along paths of successive pixels, where the paths are taken to avoid inconsistent pixels. When the SNR of the original wrapped phase is relatively high, this category of algorithms can unwrap the wrapped phase successfully. The effect of inconsistencies is distributed in a local narrow region. However, in the case of a noisy image, it sometimes creates unnatural, unintended gaps of unwrapped phase with the theoretical error of $2 n \pi$ radians, where $n$ is an integer number. This is a serious problem for quantitative evaluation of twodimensional optical distance.

The other type of algorithm is based on the leastsquares approach [15-21], in which the rewrapped result is not identical to the original wrapped phase. In these algorithms, the unwrapped phase is defined in a way that minimizes the difference of phase between the rewrapped phase and the wrapped original phase. This problem is considered to be described by a solution of the Poisson equation [18]. By appending symmetrical images outside the original image, we can assume the Neumann condition as a boundary condition $[18,20,22]$. Then we can apply the Fourier transform or discrete cosine transform (DCT) to facilitate faster computation $[20,21]$. Another method, singularity-spreading phase unwrapping (SSPU), distributes the inconsistencies [23]. It is also classified into the same type. In the computations of these methods, the unwrapped phase maps do not contain any continuous phase gaps, which had commonly appeared in the path-following methods. The path dependency, which is considered as an error or a consequence of the inconsistencies, is spread throughout the whole domain in order to avoid any large, localized errors $[6,12]$. The distributed error in the unwrapped result is considered acceptable, if it is smaller than the noise level of the wrapped noisy data in the measurement. However, the magnitude of error depends on distribution of the singular points that appear around inconsistent phase jumping segments.

In this paper, we propose a new method to compensate the inconsistencies and to confine the effect of each one in a local region. The unwrapped result of this method is similar to that of the methods based on the least-squares methods; i.e., the rewrapped result is not identical to the original phase map. However, the unwrapping is carried out along successive pixels, similar to the path-following methods. The algorithm is based on a combination of three approaches, which are named RC, USP, and VSP in this paper. The first is the rotational compensator (RC). The idea of the compensator is proposed in [23], as the singular points are spread by an iterative procedure. In our method, it can be evaluated directly without any iteration. However, the accuracy is not much improved by only the RC itself. The other two, unconstrained singular point (USP) positioning and virtual singular point (VSP), are additional approaches in order to improve accuracy. The purpose of USP is to confine the effect of the compensator to the smaller region and to determine the dipole pairs. The last approach is VSP, which is located outside the measured area in which the wrapped phase is obtained. These details are shown in Section 2 together with a comparison of past studies. The applicability of the proposed algorithm is demonstrated by numerical examples in Section 3 . Finally, the conclusion is shown in Section 4 .

\section{Phase Unwrapping Algorithm}

\section{A. Singular Point}

The wrapped phase distribution, $\Phi$, which is estimated from the fringe pattern, is defined at discrete points called pixels. It is a real-valued distribution between $(-\pi, \pi]$ radians as the principal value of an unbounded phase. Phase unwrapping in the pathfollowing type is normally carried out by comparisons of adjoining pixels. When the true, unbounded phase distribution is continuous, then, because the difference between the phases of adjoining pixels is sufficiently small, it does not exceed a half-cycle. This condition is considered as the sampling theorem. When the difference is larger than a half-cycle, the wrapped phase is shifted one cycle, so that the shifted difference is again smaller than a half-cycle. This shifting operation is the same as the wrapping operation used to obtain the principal value of the unbounded phase. The wrapping operator is defined as follows:

$$
\Phi=\mathcal{W}\{\phi\} \triangleq \phi-\operatorname{Int}\left[\frac{\phi}{2 \pi}\right] 2 \pi
$$

where $\phi$ expresses the unbounded phase and Int] means a function that returns the nearest integer. The difference vector between adjoining pixels is defined using the wrapping operator $\mathcal{W}\{\}$ as follows:

$$
\boldsymbol{g}\left(\boldsymbol{r}, \boldsymbol{r}^{\prime}\right) \triangleq \mathcal{W}\left\{\Phi\left(\boldsymbol{r}^{\prime}\right)-\Phi(\boldsymbol{r})\right\} \hat{\boldsymbol{s}}\left(\boldsymbol{r}^{\prime}-\boldsymbol{r}\right),
$$

where $r, r^{\prime}$, and $\hat{s}$ show the position of the pixel of interest, position of the adjoining pixel, and unit vector of direction $\boldsymbol{r}^{\prime}-\boldsymbol{r}$, respectively. When the pixels satisfy the sampling theorem, because the integral of the phase difference is independent of choice of the path, then the integral of $g$ along a closed path $c$ is zero:

$$
\oint_{c} g \cdot \hat{s} \mathrm{~d} l=0
$$


It should be noted that the closed path $c$ is a polygon with which line elements are along the grid because both $\boldsymbol{g}$ and $\hat{\boldsymbol{s}}$ are piecewise constant functions between two adjoining pixels. When we take the polygon-shaped path, the integral can be considered as the sum of discrete elements.

In contrast to Eq. (3), when some pixels violate the sampling theorem, the integral can take a nonzero value:

$$
\oint_{c} \boldsymbol{g} \cdot \hat{s} \mathrm{~d} l=2 \pi \sum_{k} m_{k}=2 \pi M, \quad m_{k} \in\{-1,0,1\}
$$

where the right-hand side, $M$, corresponds to a sum of the number of segments not satisfying the sampling theorem, and $m_{k}$ is the residue, the detail of which is shown below. Consider one of the smallest closed paths consisting of four pixels aligned to a square shape and evaluated in a counterclockwise direction, i.e., along the path of successive order of points $(i, j),(i+1, j),(i+1, j+1),(i, j+1)$, and $(i, j)$. We refer to this as the "elementary loop" in the later discussion. In the case where the wrapped phases are $\left(\Phi_{i j}, \Phi_{i+1, j}, \Phi_{i+1, i+1}, \Phi_{i, j+1}\right)=(0, \pi / 2, \pi,-\pi / 2)$, because all the shifted differences are $\pi / 2$, then the sum of them is $2 \pi$, which corresponds to the integral of the shifted difference. This means that either one or three of the segments do not satisfy the sampling condition, and there is a singular point (SP) with a positive residue in the area surrounded by the closed path. In the path-following methods, the SPs are positioned at the center of four pixels. In the case where the wrapped phases have opposite signs, a SP with a negative residue is found. In [24], it is shown that the residue defined as the integral normalized by $2 \pi$ of any elementary loop is always $-1,0$, or +1 .

When we take some longer closed path that includes SPs with positive residues and the same number of SPs with negative residues, because the integral is equal to zero, the unwrapping is carried out successfully. Most of the phase unwrapping algorithms of the path-following type [9-14] are based on this approach. In these methods, branch cuts, which are lines between SPs with different signed residues, are first introduced in a way that minimizes the total length of each branch cut using various approaches, such as the simulated annealing method $[11,13]$, the Hungarian algorithm [10], the minimum cost flow of network [12], and the genetic algorithm [14]. After that, the unwrapping is carried out so that the integration path does not cross the branch cuts. It is noteworthy that because the border of the measurement area can be considered as sinks of any SPs with arbitrary residue in the methods based on the pathfollowing approaches, there is no problem even in the case where the number of positive SPs in the whole measurement area and that of negative ones are different. In contrast, in the methods based on the leastsquares method, there is no such feature to cancel the effects of unbalanced SPs. If the measurement area is infinite, because the integral along the closed infinity path must be zero, the number of positive SPs and that of the negative SPs is always balanced. Otherwise, in order to establish a balance, some SPs are placed outside the area. The RC itself, shown below, has similar properties of the methods based on the least-squares method. As described in later subsections, however, it can be solved by appending virtual SPs outside the area so that the number of SPs is balanced.

\section{B. Rotational Compensator}

Yamaki and Hirose proposed the idea of introducing a compensator to cancel singularity [23]. In their method, SSPU, an original SP with residue $m$ is substituted by four fractional SPs located at adjoining points with residue $m / 4$. At this time, four compensators of $-(2 \pi m) / 4$ are appended to compensate the singularity. In the next step, the four new SPs are distributed again together with appending compensators. Repeating these procedures, the intensity of residue decreases and the SPs spread to the border of the measurement area. After the fractional residues become sufficiently small, unwrapping is carried out along successive pixels with the addition of the compensators. In the example in the previous section, the compensators of $-(2 \pi) / 4$ are appended for each segment of the path, so that the total of the wrapped phases becomes 0 , which is not singular. As a consequence of this operation, new inconsistencies appear along paths, including the segments, where each of them belongs in the points adjacent to the elementary loop, and the other compensators are evaluated for the new inconsistencies. By contrast, in our approach, the compensators are evaluated directly without any iteration.

According to the Helmholtz theorem [25], any vector is represented by the sum of two kinds of vectors: i.e., an irrotational vector that is a gradient of some scalar potential and a rotational vector that is a rotation of a vector potential. Because the unwrapped phase $\phi$ must be a scalar field, the difference vector $g$ in Eq. (2) satisfies the following equation:

$$
g=\nabla \phi+\nabla \times A .
$$

Applying the Stokes theorem to an integral of the rotation of the above equation over a domain enclosed by a path $c$, we obtain the following relation:

$$
\oint_{c} g \cdot \hat{s} \mathrm{~d} l=\oint_{c} \nabla \times \boldsymbol{A} \cdot \hat{s} \mathrm{~d} l .
$$

Comparing this equation with Eq. (4), we find that the source of the singularity is the rotation of $A$ :

$$
\oint_{c} \nabla \times \boldsymbol{A} \cdot \hat{\mathrm{s}} \mathrm{d} l=2 \pi \sum_{k} m_{k} .
$$

Because this relation is satisfied even for any elementary loop, the vector potential is considered as 
the superposed result of each vector potential corresponding to each SP:

$$
\boldsymbol{A}=\sum_{k} \boldsymbol{A}_{k}
$$

Rearranging Eqs. (5), (6), and (8), we obtain the difference of the unwrapped phase between two points as follows:

$\phi\left(\boldsymbol{r}^{\prime}\right)-\phi(\boldsymbol{r})=\int_{\boldsymbol{r}}^{r^{\prime}} \nabla \phi \cdot \hat{\boldsymbol{s}} \mathrm{d} l=\int_{\boldsymbol{r}}^{\boldsymbol{r}^{\prime}}\left(\boldsymbol{g}-\sum_{k} \nabla \times \boldsymbol{A}_{k}\right) \cdot \hat{\boldsymbol{s}} \mathrm{d} l$.

This equation shows that the singularity of the wrapped difference vector $g$ is compensated by the rotation of the vector potential $\boldsymbol{A}_{k}$. Thus, we refer to the integral of rotation of $\boldsymbol{A}_{k}$ as the RC.

In order to evaluate each $\mathrm{RC}$, let us consider the cylindrical coordinate of $(R, \theta, z)$, where the $k$ th SP is located at the origin. Each vector potential satisfies the following relation:

$$
\oint_{c} \nabla \times A_{k} \cdot \hat{s} \mathrm{~d} l=2 \pi m_{k} .
$$

Each source of singularity has an axial symmetry where every component of the vector $\boldsymbol{A}_{k}$ is represented only in terms of a function of radial distance $R$ as $A_{k}=\left(a_{R}(R), a_{\theta}(R), a_{z}(R)\right)$. Furthermore, the $z$ component of the unit tangential vector $\hat{s}$ is zero. Therefore, the integrand is reduced as follows:

$$
\nabla \times \boldsymbol{A}_{k} \cdot \hat{\boldsymbol{s}}=\frac{\partial a_{z}}{\partial R} \boldsymbol{e}_{R} \times \boldsymbol{e}_{z} \cdot \hat{\boldsymbol{s}}=-\frac{\partial a_{z}}{\partial R} \boldsymbol{e}_{R} \cdot \hat{\boldsymbol{n}},
$$

where $\boldsymbol{e}_{R}$ and $\boldsymbol{e}_{z}$ are unit vectors for the $R$ axis and $z$ axis, respectively, and $\hat{n}$ denotes the outward normal unit vector to the path $\left(\hat{\boldsymbol{n}}=\hat{\boldsymbol{s}} \times \boldsymbol{e}_{z}\right)$. Because the integrand in the left-hand side in Eq. (10) is a regular function except at the origin, the integral path can be modified to an arbitrary path that surrounds the origin. In the case where the path is taken as a circular path with radius $R$, because $\hat{\boldsymbol{n}}$ is identical to $e_{R}$, the left-hand side of Eq. (10) is readily obtained as $-2 \pi R\left(\partial a_{z} / \partial R\right)$. Therefore, the partial derivative is represented as

$$
\frac{\partial a_{z}}{\partial R}=-\frac{m_{k}}{R} .
$$

Consequently, the integral along an arbitrary closed path expressed in Eq. (10) is rewritten as

$$
\oint_{c} \nabla \times \boldsymbol{A}_{k} \cdot \hat{s} \mathrm{~d} l=\oint_{c} \frac{m_{k}}{R} \boldsymbol{e}_{R} \cdot \hat{\boldsymbol{n}} \mathrm{d} l .
$$

The integral along a segment of which ends are $\boldsymbol{r}_{1}$ and $\boldsymbol{r}_{2}$ is evaluated by taking the closed path $c$ as shown in Fig. 1. Because there is no $\mathrm{SP}$ in the domain

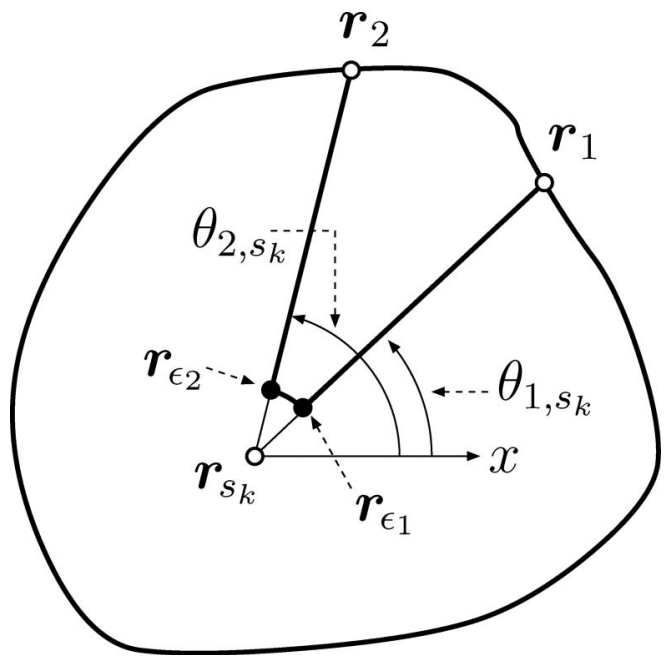

Fig. 1. Integration path to evaluate the RC.

surrounded by the closed path $\boldsymbol{r}_{1}, \boldsymbol{r}_{2}, \boldsymbol{r}_{\mathrm{\epsilon}_{1}}$, and $\boldsymbol{r}_{\mathrm{\epsilon}_{2}}$, the integral along the closed path vanishes. Each of the integrals along the two straight lines also vanishes because the normal unit vector is perpendicular to the $\boldsymbol{e}_{R}$. Thus, the compensator for the SP that is the integral along the segment may be estimated from the integral along the semicircle as follows:

$$
\begin{gathered}
C_{k}\left(\boldsymbol{r}_{1}, \boldsymbol{r}_{2}\right) \triangleq-\int_{\boldsymbol{r}_{1}}^{r_{2}} \nabla \times \boldsymbol{A}_{k} \cdot \hat{\boldsymbol{s}} \mathrm{d} l=-\int_{\boldsymbol{r}_{1}}^{r_{2}} \frac{m_{k}}{R} \boldsymbol{e}_{R} \cdot \hat{\boldsymbol{n}} \mathrm{d} l \\
=\int_{\boldsymbol{r}_{\mathrm{\epsilon} 1}}^{r_{\mathrm{\epsilon} 2}} \frac{m_{k}}{R} \boldsymbol{e}_{R} \cdot \hat{\boldsymbol{n}} \mathrm{d} l=\int_{\theta_{1, s_{k}}}^{\theta_{2, s_{k}}} \frac{m_{k}}{\epsilon} \boldsymbol{e}_{R} \cdot\left(-\boldsymbol{e}_{R}\right) \epsilon \mathrm{d} \theta \\
=-m_{k}\left(\theta_{2, s_{k}}-\theta_{1, s_{k}}\right),
\end{gathered}
$$

where $\theta$ denotes the unbounded azimuthal angle from the $x$ axis and also $\theta_{2, s_{k}}>\theta_{1, s_{k}}$.

Taking $\boldsymbol{r}_{1}$ and $\boldsymbol{r}_{2}$ in Eq. (14) as the adjoining pixels $\boldsymbol{r}$ and $\boldsymbol{r}^{\prime}$, respectively, we can unwrap the wrapped phase by applying the wrapped difference vector $g$ and the compensators $C_{k}$ as follows:

$$
\phi\left(\boldsymbol{r}^{\prime}\right)=\phi(\boldsymbol{r})+\boldsymbol{g}\left(\boldsymbol{r}, \boldsymbol{r}^{\prime}\right) \cdot \hat{\boldsymbol{s}}\left(\boldsymbol{r}^{\prime}-\boldsymbol{r}\right)+\sum_{k} C_{k}\left(\boldsymbol{r}, \boldsymbol{r}^{\prime}\right) .
$$

The compensator of a SP spreads throughout the whole measurement area. It seems to share a similar nature with the methods based on the least-squares approach, in which the effect of singular points also spreads [6]. However, it is found that the compensator decreases with increasing $R$ because the distance between $\boldsymbol{r}^{\prime}$ and $\boldsymbol{r}$ is always kept constant at one pixel width. This characteristic is similar to path-following methods, in which the effect of a singular point is confined into the region that surrounds the branch cut.

\section{Unconstrained Singular Point Positioning}

The RC can remove the inconsistency by the cancellation of the singularity. However, it introduces an undesired distortion of phase in a wide area. In order 
to limit the distorted area in the narrower region around the SP, we propose an USP.

The RC, $C_{k}$, defined in Eq. (14) cancels a single SP. The effect of the single SP that appears in the kernel of the integral can be considered as a monopole, which is proportional to the reciprocal of the distance $R$ :

$$
E_{\text {monopole }}=-\frac{1}{R} e_{R}
$$

Let us consider the case in which another SP with an opposite-signed residue is located near the original one. This case corresponds to a dipole, which is often found in the literature of electromagnetics. The effect of the dipole in two-dimensional space is evaluated as follows:

$$
\boldsymbol{E}_{\mathrm{dipole}}=-\frac{1}{R^{2}}\left(2\left(\boldsymbol{d} \cdot \boldsymbol{e}_{R}\right) \boldsymbol{e}_{R}-\boldsymbol{d}\right)
$$

where $\boldsymbol{d}$ is the difference vector from the negative SP to the positive SP, and the origin is taken at the center of the two SPs. The decay of effect induced by a dipole is faster than that by a monopole. It is known that the distance of the nearest SP with an oppositesigned residue is shorter than that with a samesigned residue from analysis of an actual noisy experimental wrapped phase [11]. Therefore, if every SP belongs to a dipole, the effect of compensator is $|d| / R$ times smaller than that of a single compensator, it means the effect is confined in a local region around the dipole.

The SP is generally positioned at the center point of an elementary path that consists of four pixels aligned with a square shape, and the minimum $|\boldsymbol{d}|$ is limited to the pixel size. If we can obtain the position accurately and if the distance of the dipole is closer than the pixel size, the effect of the compensator is limited to a narrower region. Ideally, the affected region approaches that in the method of the branch cut.

The approach to find an SP position that is not constrained at the center point at an elementary loop is as follows. It is found from Eq. (14) that the compensator of a segment is represented as the difference of the azimuthal angle between the ends of the segment. This means that we can consider the following model of wrapped phase maps with singularities:

$$
\begin{gathered}
\Phi(\boldsymbol{r})=\mathcal{W}\left\{m \theta\left(\boldsymbol{r}, \boldsymbol{r}_{s}\right)+\bar{\phi}+\delta \phi(\boldsymbol{r})\right\}, \\
(m \in\{-1,+1\}),
\end{gathered}
$$

where $\bar{\phi}$ and $\delta \phi(r)$ are a phase average and a nonsingular phase fluctuation, respectively. Let us consider the following wrapped difference between adjacent pixels:

$$
\Delta \triangleq \mathcal{W}\left\{\mathcal{W}\left\{\Phi^{\prime}-\Phi\right\}-m\left(\theta^{\prime}-\theta\right)\right\},
$$

in which the prime mark means quantities at adjacent pixels. Substituting Eq. (18) to the difference and applying a characteristic of the wrapping opera- tor as $\mathcal{W}\{\mathcal{W}\{\Psi\}\} \equiv \mathcal{W}\{\Psi\}$, we can reduce the wrapped difference to

$$
\Delta=\mathcal{W}\left\{\delta \phi^{\prime}-\delta \phi\right\} .
$$

Because this relation suggests that a solution minimizing $|\Delta|$ is equivalent to a solution minimizing $\left|\mathcal{W}\left\{\delta \phi^{\prime}-\delta \phi\right\}\right|$, then when we can assume the unknown phase fluctuation $\delta \phi$ is small, the problem to determine $\boldsymbol{r}_{s}$ is reduced to the problem to find the solution with minimized $|\Delta|$ in Eq. (19). Thus, we define the problem by means of a nonlinear leastsquares problem as follows:

$$
\begin{gathered}
\operatorname{minimize} \sum_{l=0}^{3} \Delta^{2}=\sum_{l=0}^{3}\left(\mathcal{W}\left\{\mathcal{W}\left\{\Delta \Phi_{l}\right\}-m \Delta \theta_{l}\left(\boldsymbol{r}_{s}\right)\right\}\right)^{2}, \\
\Delta \Phi_{l} \triangleq \Phi_{l+1}-\Phi_{l}, \quad \Phi_{4}=\Phi_{0}, \\
\Delta \theta_{l} \triangleq \theta_{l+1}\left(\boldsymbol{r}_{s}\right)-\theta_{l}\left(\boldsymbol{r}_{s}\right)>0, \quad \theta_{4}=\theta_{0}+2 \pi,
\end{gathered}
$$

where $l$ denotes the identifier of a segment or a pixel in an elementary loop, shown in Fig. 2, and $l \in\{0, \ldots, 3\}$. Because this nonlinear minimization problem is difficult to solve analytically, we applied a numerical method based on a genetic algorithm [26].

\section{Virtual Singular Point}

As described in the previous subsection, the compensator effect of a dipole decreases rapidly with an increasing distance from the dipole. However, because the measurement area is finite, there are some isolated SPs or SPs of dipoles with long distances. The isolated SPs spread error throughout the entire measurement area. When we put virtual SPs having an opposite sign of the isolated SPs outside the area, the virtual SPs and the isolated SPs make dipoles, and then the error may be reduced. In the case where the isolated SP is located near the border of the area,

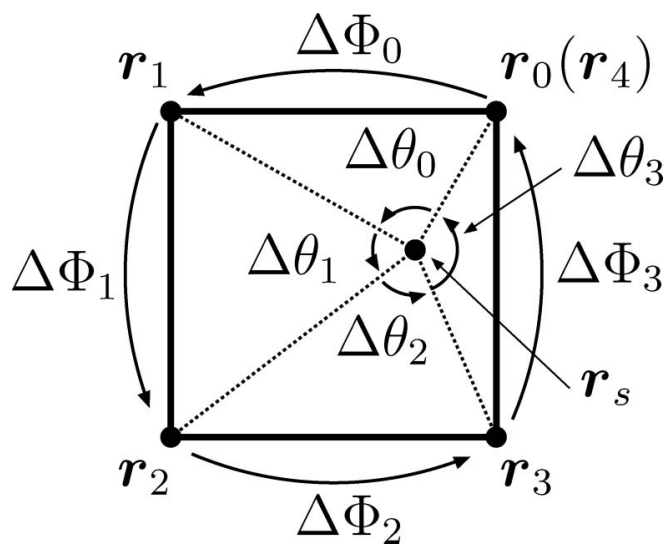

Fig. 2. Elementary path including an USP: $\Delta \theta_{l}$ means the facing angle of the side with the ends $r_{l}$ and $r_{l+1}$ and $\Delta \Phi_{l}$ means the difference of the wrapped phases. 
the virtual SP is put at the symmetrical point to the border outside the area so that the center of the dipole is located just on the border. It should be noted that there is no empirical basis to take the virtual SP at the symmetrical point. It is intuitively guessed that the probability of a virtual SP to be located in a region far from the border is small. In contrast, the case where a virtual SP is located just on the border is also rare. If we could know the probability distribution of the size of dipoles that are located around the border, we would be able to statistically determine the position. Gutmann and Weber [13] used the distance distribution of SPs inside the whole measurement area in their paper, and this is applied to the search for branch cuts. However, we do not apply their method because the distance distribution may depend on the condition around the local area and it may be smaller than one pixel width under the use of the unconstrained SP positioning.

To find isolated SPs near the border, we attempt the following approach; a schematic example is shown in Fig. $\underline{3}$.

1. Preparation:

Mark all SPs as "isolated."

For each SP, locate the symmetrical point to the nearest border point. The symmetrical point is defined and marked as a virtual SP candidate with an opposite sign. The virtual SP candidates are shown as the end of the dashed arrows in Fig. 3(a).

2. Find the nearest singular point:

For every SP with the mark "isolated," find the nearest SP with an opposite sign among SPs marked as "isolated," also including the virtual SP candidate corresponding to the original SP.

3. Virtual SP determination:

If the nearest SP is the virtual SP candidate, the original SP is marked as "dipole with virtual," and the virtual SP candidate is marked as "virtual." The pair of them is shown as an encircled pair with single-headed arrows, in Fig. 3(b).

4. Dipole determination:

For each positive SP with the mark "isolated," if the nearest negative SP has no other positive SP that is closer than the original one, then both of the SPs are marked as "internal dipole." The pair of them is depicted as an encircled pair with double-headed arrows, as shown in Fig. 3(b). The virtual candidates corresponding to these SPs, which have ticked symbols in Fig. 3(b), are removed from the virtual candidates list.

5. Repetition:

Repeat procedures 2 to 4 , until no more SPs marked with "isolated" are found. After these

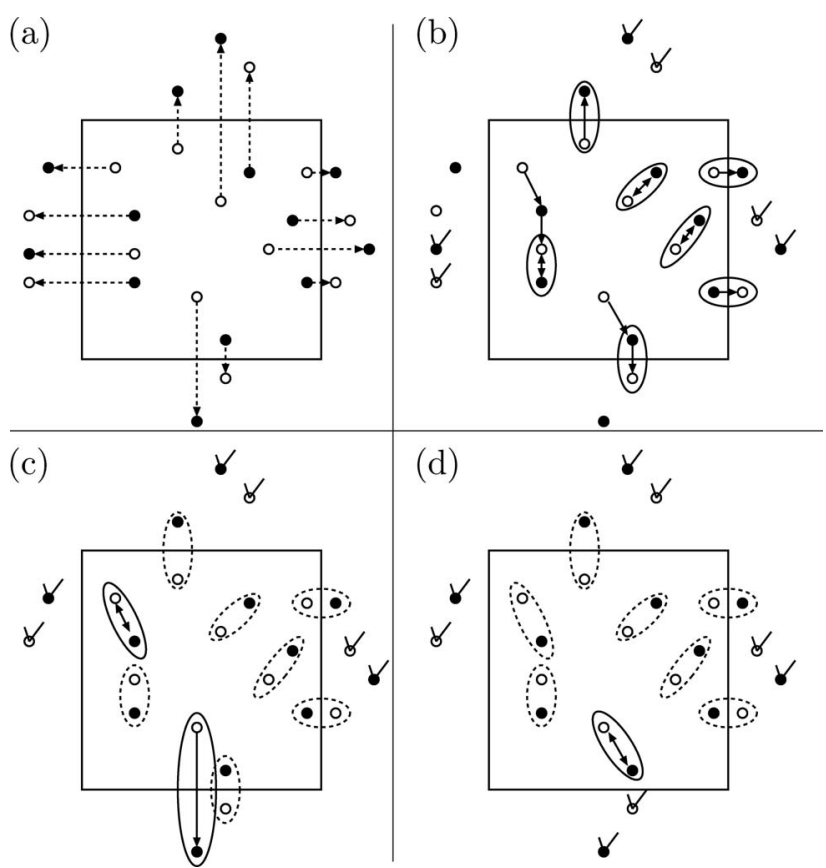

Fig. 3. Determination of dipole pairs. (a) Definition of the virtual SP candidates: Square frame shows the measurement area. Open and solid circles show positive and negative SPs, respectively. Each dashed arrow shows the correspondence between original and virtual SP. (b) Nearest SP search and pairing in the first iteration: Each arrow shows the nearest opposite-signed SP. The SPs encircled are paired as dipoles. The ticked virtual candidate is removed from the list of candidates in subsequent steps. (c) Second iteration: SPs enclosed with dashed ellipses have been already paired. (d) Elimination of removable virtual SP pairs.

steps, all SPs are marked as "internal dipole," "dipole with virtual," or "virtual." See Fig. 3(c).

6. Elimination of removable VSP pair:

For each SP marked "dipole with virtual," if the nearest opposite-signed SP within the SPs located inside the border is also marked as "dipole with virtual," let us examine whether this pair can become a new pair. If twice the distance of the new pair is shorter than the sum of the distances between the SP and the current partner with the mark "virtual," the total branch-cut length is shorter than the current pairs. In this case, the new pair is coupled and the marks of the old partners "virtual" are removed. This elimination is shown in Fig. $3(\mathrm{~d})$.

The idea of step (6) is found in [27].

\section{Numerical Example of Phase Unwrapping Noisy, Wrapped Phase Map}

\section{A. Unwrapping Simulation for Known Phase Map}

True phase in experimental results is unknown. Using a wrapped phase map that is a result of a known unwrapped phase map instead of a wrapped phase map obtained by an experiment, we can examine the characteristics of unwrapping methods. 
The prepared original continuous phase map is a noisy phase map with a constant gradient; the image size is 100 pixels $\times 100$ pixels, the gradient is $(0.1,-0.1)$ cycle/pixel, and the noise has a normal distribution with 0.15 cycle standard deviation. The phase map is an unlimited function as a true solution. It is shown in Fig. 4(a) together with its wrapped phase. The wrapped phase is the input for unwrapping algorithms. In this wrapped phase, the numbers of positive and negative SPs are 453 and 456, respectively, overall comprising almost $9 \%$ of the number of all pixels, the distribution of which is shown in Fig. 5. It should be noted that there are three unbalanced SPs, which cannot make pairs, even when coupling with a longer distance is permitted. The magnified distribution of SPs around the region that includes the VSP at the bottom right border is shown in Fig. 6. In this figure, the USPs are determined by the approach described in Subsection 2.C, and the VSP found by the approach described in Subsection 2.D are also shown. The distances of some USP dipoles are shorter than those of the original dipoles, which consist of the constrained SPs.

The distribution of distances between each positive SP and its nearest negative SP is shown in Fig. 7. From the distribution of the constrained SPs that are located in the center of the elementary loops, it is found that most of the distances are concentrated at 1 or $\sqrt{2}$. In contrast, the average distance of USPs is significantly shorter than that of constrained SPs. This suggests that the effect of the compensator with the use of USPs is confined in a narrower region than with constrained SP positioning.

In order to compare the characteristics, we applied several algorithms: the Goldstein path-following method [8], the least-squares method with discrete cosine transform (LS-DCT) [21], SSPU [23], and the proposed algorithm with several options in this article. Some results of the unwrapped phase maps together with rewrapped phases are shown in Figs. $4(\mathrm{~b})-4(\mathrm{~d})$. Some continuous phase gaps are found in the unwrapped phase map obtained by the Goldstein method in Fig. 4(b). Although the unwrapped phase maps by both the LS-DCT and the proposed method [Fig. 4(c) and 4(d)] have no phase gap, we found a smaller number of stripes in the rewrapped phase than in the original wrapped phase. This indicates that the gradient of the unwrapped phase was underestimated. Although unwrapped or rewrapped phase maps for the other algorithms are not shown as images, they show similar properties.

To demonstrate a quantitative comparison, gradients of the unwrapped phase maps are shown in Table 1 . In the table, the gradients are obtained by fitting to a planar function; i.e., $\tilde{\phi}(\boldsymbol{r})=\nabla \tilde{\phi} \cdot \boldsymbol{r}+\tilde{\phi}_{0}$, and $\sigma$ denotes the mean residual that is defined as a square root of a mean square residual from $\tilde{\phi}$. Even in the original data, the $\sigma$ is not equal to zero because the original data contain the noise with the given standard deviation. The errors of gradient and mean residual, $\Delta(\nabla \tilde{\phi})$ and $\Delta \sigma$, are estimated as the normalized difference between the unwrapped result and the original one, where the normalizing factor is the reciprocal of the original one. From the table it is found that the gradients are underestimated by all of the algorithms. The best result in terms of $\Delta(\nabla \tilde{\phi})$ and $\Delta \sigma$ is found in Table $1(\mathrm{~g})$, in which all of the proposed approaches (the RC, USP, and VSP) are applied. The Goldstein method in (a) has a large $\Delta \sigma$. The reason of this is evident from the unwrapped image shown in Fig. $4(\mathrm{~b})$; there is a continuous set of (a)

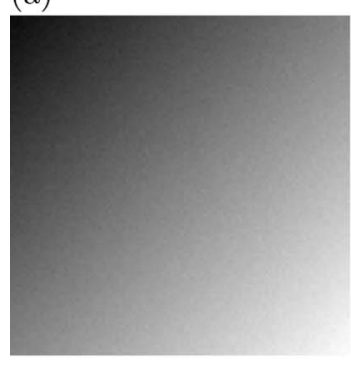

(c)

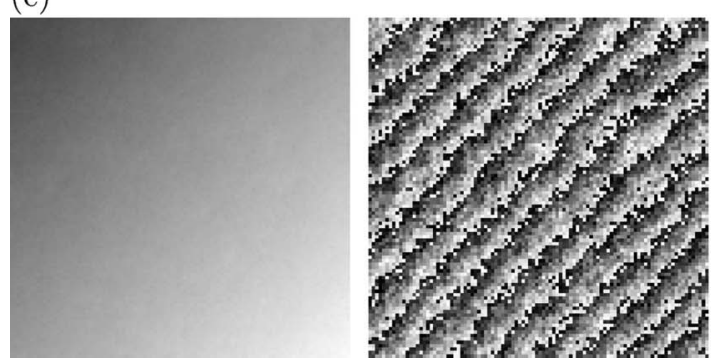

(b)

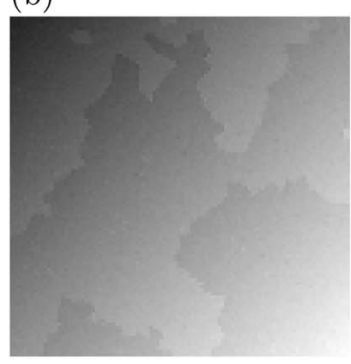

(d)

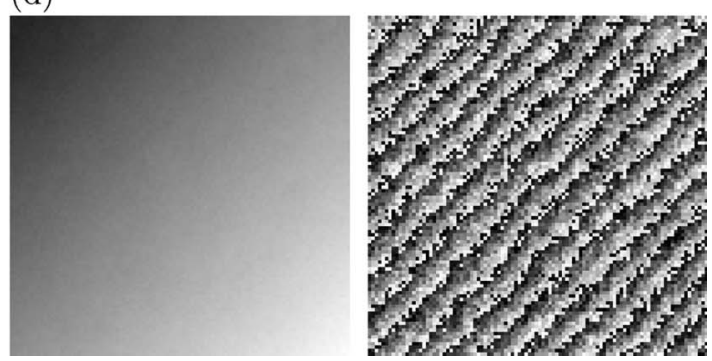

Fig. 4. Unwrapped and rewrapped phase map: In each figure, the left-hand side figure shows the unwrapped or original phase map, where the phase increases with the increase of brightness, and the right-hand side figure shows a rewrapped or wrapped phase map. (a) Original phase map. (b) Goldstein path-following method. (c) LS-DCT. (d) Proposed method using all the RC, the USP, and the VSP. 


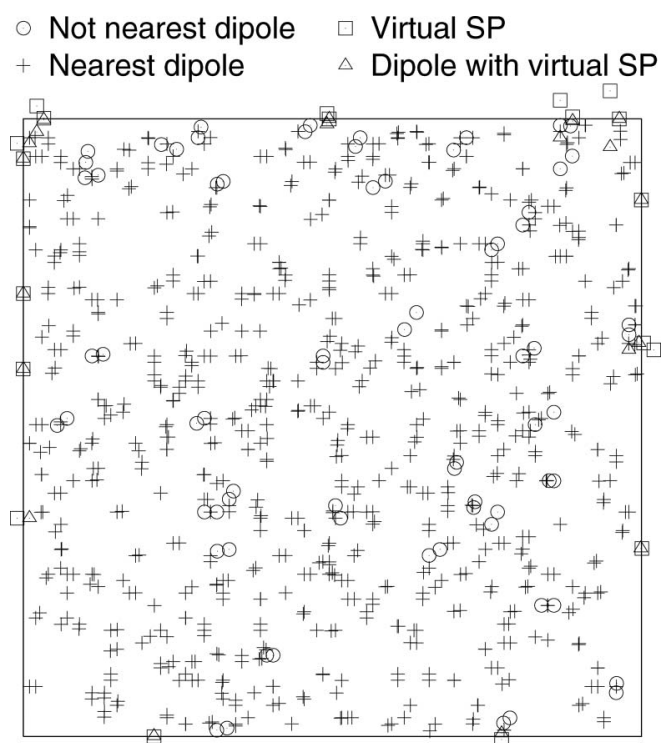

Fig. 5. Distribution of SPs: All of the SPs are coupled with another SP. In the legend, both "Nearest dipole" and "Not nearest dipole" mean that both SPs of a pair are located inside the measurement area; "Nearest" shows that the SPs are nearest each other; Alternately, pairs are formed from "Dipole with virtual SP" located inside the area and "Virtual SP" located outside the area.

gaps. From the comparison (b)-(d) in Table 1, it is found that the errors in the methods of LS-DCT, SSPU, and RC are nearly the same. Using these three methods, the inconsistencies by SPs are canceled; however, imbalance of the number of positive and negative SPs is not considered. From a comparison among (d)-(f), it seems that the VPS is more effective for improving the accuracy of the gradient, and the USP is more effective for reducing the error

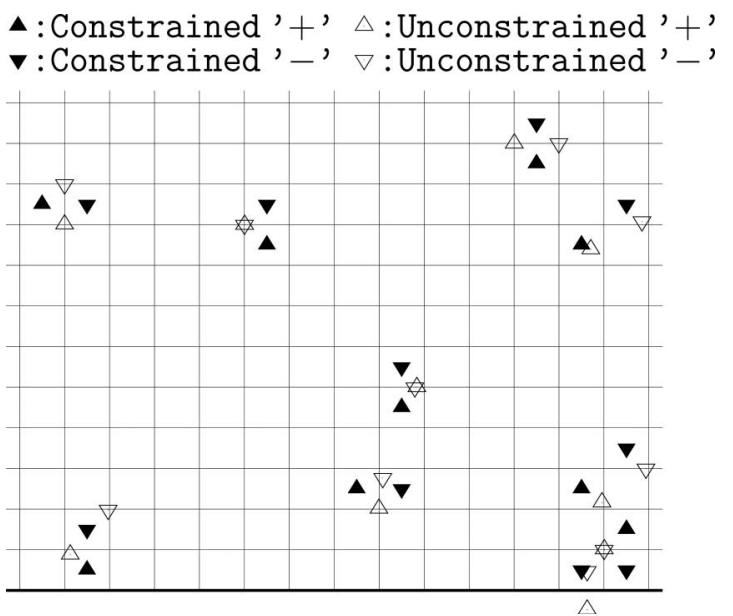

Fig. 6. SP around the bottom border: Pixels where the wrapped data are defined are located at intersections on the grid. The points denoted by solid symbols are constrained SPs located at the center of the smallest grid; i.e., the elementary loop. The points denoted by open symbols are unconstrained SPs. The regular and the inverted triangle symbols express positive and negative SPs, respectively. The thick line at the bottom is the border of the measurement area. The SPs outside the area (under the border) correspond to VSPs.

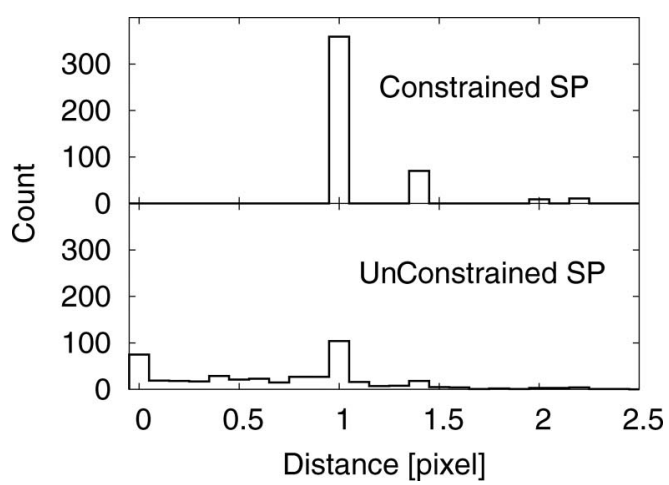

Fig. 7. Frequency histogram for distance from a positive SP to the nearest negative SP.

of the mean residual. These results can be explained by the nature of each approach. The error of the gradient shows global error, while the mean residual shows local error from the global distribution of the unwrapped phase. If isolated SPs that are not SPs containing dipoles are found in the measured area, the compensator affects the entire area, which should otherwise be confined into a local area. Because the VSP is applied to avoid the existence of such isolated SPs, the global error is reduced. On the other hand, because it is found that the USP can make dipoles with a shorter range, as shown in Fig. 7, the compensator to remove the dipole affects only a narrower region.

We consider the following two reasons why the proposed method with all the approaches still has some error. The first is the position of the VSP. As described in Subsection 2.D, there is no empirical basis to put the VSP at a symmetrical point to the nearest border. However, this factor is not critical to the error. Even if the position of a VSP differs slightly from the true SP outside the region, the VSP still makes a dipole; therefore, the effect to the global error corresponding to the error of the gradient may be small. A more significant source of error in terms of VSP is induced by incorrect coupling through the method shown in Subsection 2.D. If the detected isolated SPs are incorrect, the position of each VSP is taken at a more distant point from the true SP, and then it can induce the global error. The second source is the model to determine the wrapped phase shown in Eq. (18). If the fluctuation $\delta \phi$ is not sufficiently small, the approach to determine the USP is not suitable. This may affect the local error corresponding to the error of the mean residual.

Figure 8 shows a comparison of the required computational time. This comparison between algorithms is not strictly meaningful because the computational time depends on implementations of each algorithm. However, the computational time dependency on the measurement area size is considered independent of implementation type. In the method based on the RC, the computational time is almost proportional to $N^{4}$ with $N>200$, where $N$ denotes the one-dimensional area size in units of pixels. This trend is reasonable. In these cases, when the 
Table 1. Accuracy Comparison among Algorithms by Planar Function Fitting

\begin{tabular}{llcccc}
\hline & \multicolumn{1}{c}{ Algorithm } & Gradient $(\nabla \tilde{\phi})$ & $\Delta(\nabla \tilde{\phi})[\%]$ & $\sigma$ & $\Delta \sigma[\%]$ \\
\hline Original & $(0.1000,-0.1000)$ & $(-,-)$ & 0.149 \\
\hline (a) & Goldstein & $(0.0892,-0.0826)$ & $(-10.8,-17.4)$ & 0.425 & +184.9 \\
(c) & LS-DCT & $(0.0742,-0.0731)$ & $(-25.8,-27.0)$ & 0.179 & +20.0 \\
(d) & SSPU & $(0.0743,-0.0730)$ & $(-25.7,-27.0)$ & 0.178 & +19.7 \\
(e) & RC & $(0.0816,-0.0722)$ & $(-18.4,-27.9)$ & 0.182 & +21.7 \\
(f) & RC + USP & $(0.0860,-0.0756)$ & $(-14.1,-24.5)$ & 0.173 & +15.9 \\
(g) & RC + VSP & $(0.0870,-0.0862)$ & $(-13.0,-13.7)$ & 0.187 & +25.6 \\
\hline
\end{tabular}

overhead to the main computation is negligible, most of the time is elapsed in evaluations of compensation. The amount of evaluation time is proportional to both the number of SPs and the number of the segments of path to be compensated. Because both are proportional to the area size $\left(\propto N^{2}\right)$, the total evaluation time is proportional to $N^{4}$. In contrast, the computational time increases with $N^{3}$ in the method using LS-DCT. In this computation, we used a simple algorithm, not a fast Fourier transform, but we use a two-dimensional buffer. Through the use of the buffer, the computational time of two-dimensional cosine transforms needs only $N^{3}$ multiplications. Thus, the method based on the $\mathrm{RC}$ is more time consuming than that of LS-DCT, unfortunately.

\section{B. Unwrapping for Interferogram by Experiment}

In order to demonstrate validity of the proposed method to the actual experimental data, we applied the method to measuring the phase shift in candle flames. The result is shown in Fig. 9. The fringes, $I_{\text {obj }}$ and $I_{\mathrm{bg}}$, are obtained by a Mach-Zehnder interferometer. The fringe $I_{\mathrm{obj}}$ is a superposed result of object light passing through the candle flame upon the reference light. The fringe $I_{\mathrm{bg}}$ is a result from the same system, but there is no flame. These results are measured by a digital video camera (PixeLINK, A741). At the bottom of $I_{\text {obj }}$ and $I_{\mathrm{bg}}$, a shadow of a core of the candle is found. Because the fringe information is not obtained in the shadow, in order to exclude the shadow, we set the region of interest (ROI) with

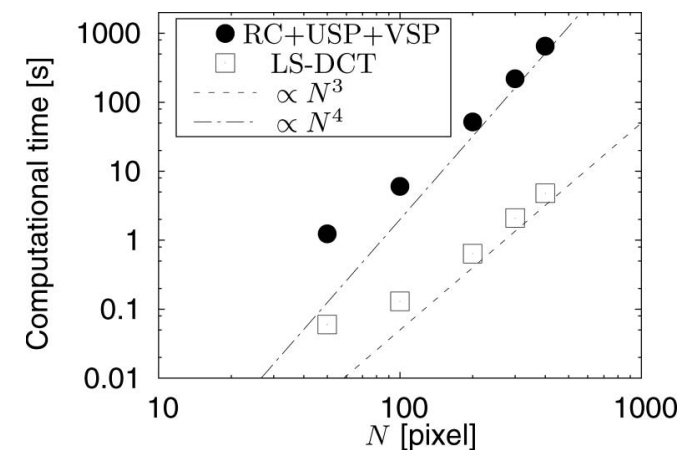

Fig. 8. Computational time: The horizontal axis $N$ denotes the one-dimensional area size in pixels. "RC + USP + VSP" shows the result by the proposed method. The computational time is measured with a PC including an Intel Core 2 DUO CPU with $2.13 \mathrm{GHz}$ clock in a single CPU operation mode.
256 pixels $\times 170$ pixels size. In this measurement we cannot set the exposure time long enough because the flame is varying in time by convection flow around the flame itself. Therefore, we set the exposure time to $1 \mathrm{~ms}$. This setting induces two difficulties: (i) the fringe has low SNR, (ii) we cannot apply the phase shift techniques $[2,4,5]$ that use several fringes with different reference lights to obtain a

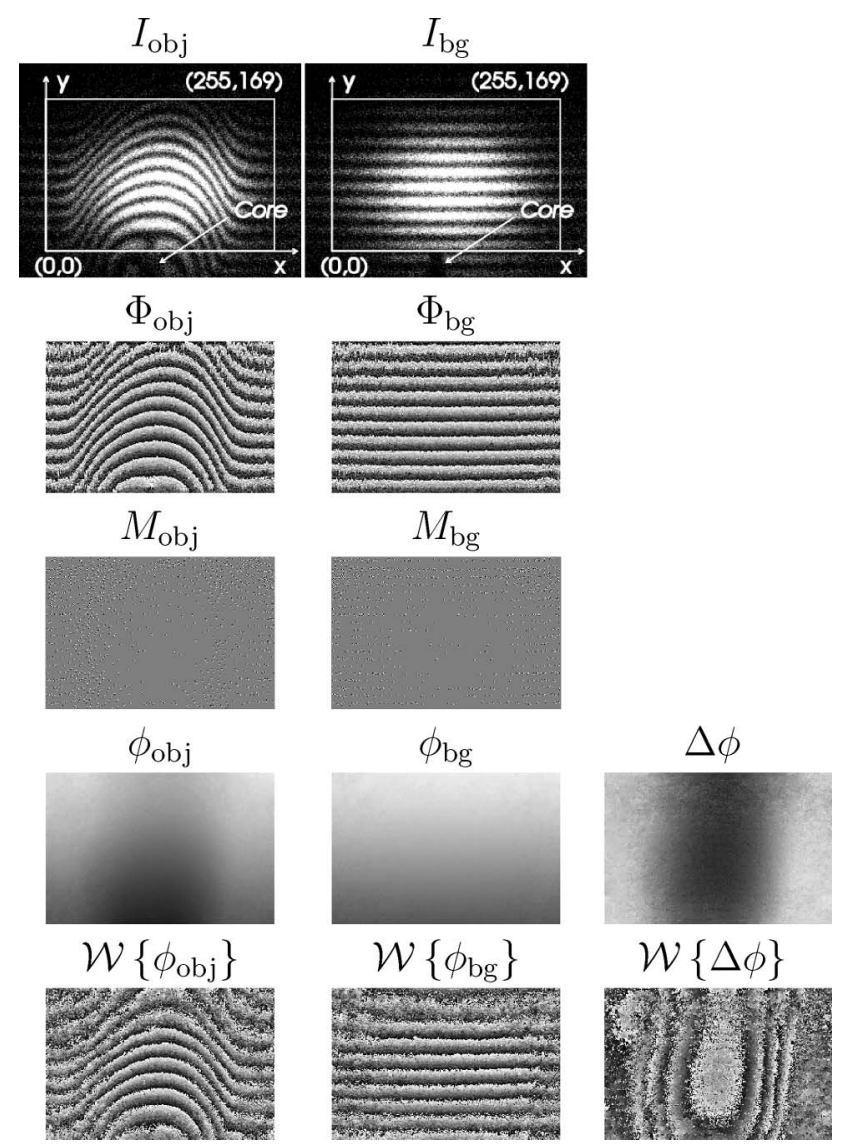

Fig. 9. Unwrapped phase of fringe by Mach-Zehnder interferometer for candle flame: The $I, \Phi, M, \phi$, and $\mathcal{W}\{\}$ show observed fringe patterns with enhancement of contrast, wrapped phase maps by Fourier domain method, distributions of SPs (positive and negative SPs are represented by white and black dots, respectively), unwrapped phase maps by the proposed method (RC, USP, and VSP), and wrapping operators, respectively. The subscripts "obj" and "bg" mean object and background, respectively. The unwrapped phase difference, $\Delta \phi=\phi_{\mathrm{obj}}-\phi_{\mathrm{bg}}$, shows the actual phase shift by the flame. 
wrapped phase. The aim of this paper is to solve the first difficulty. The second difficulty in the calculation of a wrapped phase can be solved by applying the Fourier domain method [1-3]. It is useful to obtain the wrapped phase map from a single fringe pattern; however, it contains some distortion of the map. For example, it is found around the candle core from the comparison between $I_{\text {obj }}$ and $\Phi_{\text {obj }}$. This problem cannot be solved by any unwrapping approaches. Both of the residue maps, $M_{\mathrm{obj}}$ and $M_{\mathrm{bg}}$, show that many SPs are found around the border, where the SNR is low in fringe patterns. The difference between the simulation data in the previous section and the experimental data is found in this distribution. The amount of SPs in all pixels within the ROI reaches as near as $6 \%$ in $M_{\mathrm{obj}}$ and $4 \%$ in $M_{\mathrm{bg}}$. The unwrapped results by the proposed method using all the RC, the USP, and the VSP are shown as $\phi_{\mathrm{obj}}$ and $\phi_{\mathrm{bg}}$. The actual phase shift can be evaluated by the difference between $\phi_{\text {obj }}$ and $\phi_{\mathrm{bg}}, \Delta \phi \equiv \phi_{\mathrm{obj}}-\phi_{\mathrm{bg}}$. To evaluate the characteristics of the phase-unwrapping method, the rewrapped phases, $\mathcal{W}\{\phi\}$, are also shown. From a comparison between the original wrapped phase $\Phi$ and the rewrapped phase $\mathcal{W}\{\phi\}$, we can find that the number of stripes in $\mathcal{W}\{\phi\}$ is less than that in $\Phi$, which is similar to the simulated result in the previous section. Furthermore, we can find the result with noisy distribution around the low SNR region.

We also computed the unwrapped phase by the LSDCT [21]. Although the images by the LS-DCT are not shown because of a lack of space, the number of fringes is also less than that in $\Phi$, and it is less than $\mathcal{W}\{\phi\}$ by the proposed method (RC + USP+ VSP). Figure 10 shows the comparison of $\Delta \phi$ between the RC + USP + VSP and the LS-DCT along $y=85$ (the half height in the ROI). The phase shifts around the center $(x=100 \sim 150)$ are almost 3.9 in the RC + USP + VSP and 2.7 in the LS-DCT, smaller than the phase shift around the edge. We can estimate roughly by reading the original fringe pattern $I_{\text {obj }}$. The number of fringes along $y=85$ from $x=0$ to $x=128$, it reaches almost four to five cycles. Thus, the unwrapped results in both methods are underes-

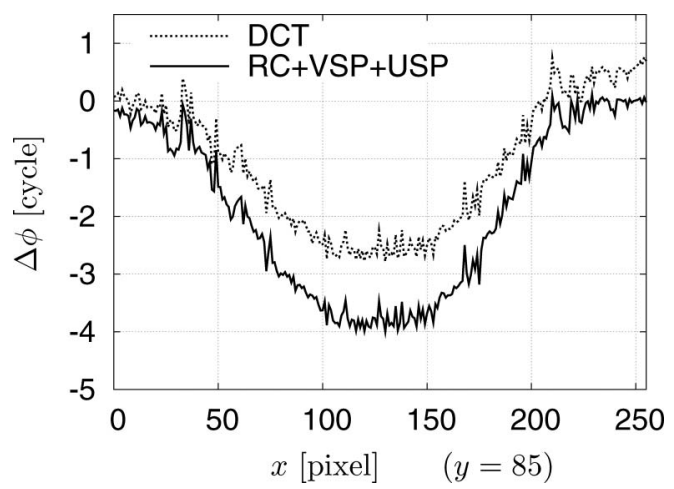

Fig. 10. Comparison of unwrapped phase differences: "RC + USP + VSP" shows the unwrapped phase difference, $\Delta \phi$, along $y=85$ in Fig. $\underline{9}$, and the "LS-DCT" shows it by the LSDCT method. timated; however, the underestimation in the $\mathrm{RC}+$ USP + VSP is smaller than that in the LS-DCT.

\section{Conclusion}

For the purpose of more accurate phase unwrapping for noisy wrapped phase maps, we propose a new method based on a combination of three approaches: RC, USP, and VSP. The RC acts to compensate the singularity of each SP for all unwrapping paths. The USP provides freedom to adjust the SP positioning in order to improve the accuracy of compensation. Because it can make some dipoles that have shorter distances than the pixel size, the undesired, longer effect of the compensator is suppressed. The VSPs for unpaired, isolated SPs are taken outside the area to confine the effect of the compensator in local narrow regions around SPs. In the comparisons of several methods of phase unwrapping through both a numerical simulation and an analysis of the experimental fringe pattern, the proposed method demonstrates better accuracy of unwrapping (compared to the Goldstein method, the LS-DCT, and SSPU), although it does not eliminate the phase error with underestimation. One drawback of the proposed method is a greater computational time requirement, compared to the other methods.

This research was supported in part by the Japan Society for the Promotion of Science, Grant-in-Aid for Scientific Research (C), 21560197, 2009.

\section{References}

1. M. Takeda, H. Ina, and S. Kobayashi, "Fourier-transform method of fringe-pattern analysis for computer-based topography and interferometry," J. Opt. Soc. Am. 72, 156-160 (1982).

2. K. E. Perry, Jr., and J. McKelvie, "A comparison of phase shifting and Fourier methods in the analysis of discontinuous fringe patterns," Opt. Lasers Eng. 19, 269-284 (1993).

3. E. Cuche, P. Marquet, and C. Depeursinge, "Spatial filtering for zero-order and twin-image elimination in digital off-axis holography," Appl. Opt. 39, 4070-4075 (2000).

4. J. H. Bruning, D. R. Herriott, J. E. Gallagher, D. P. Rosenfeld, A. D. White, and D. J. Brangaccio, "Digital wavefront measuring interferometer for testing optical surfaces and lenses," Appl. Opt. 13, 2693-2703 (1974).

5. B. Breuckmann and W. Thieme, "Computer-aided analysis of holographic interferograms using the phase-shift method," Appl. Opt. 24, 2145-2149 (1985).

6. D. C. Ghiglia and M. D. Pritt, Two-Dimensional Phase Unwrapping: Theory, Algorithms, and Software (Wiley, 1998).

7. R. Vandenhouten and R. Grebe, "Phase reconstruction and unwrapping from holographic interferograms of partially absorbent phase objects," Appl. Opt. 34, 1401-1406 (1995).

8. R. M. Goldstein, H. A. Zebker, and C. L. Werner, "Satellite radar interferometry: two-dimensional phase unwrapping," Radio Sci. 23, 713-720 (1988).

9. J. M. Huntley, "Noise-immune phase unwrapping algorithm," Appl. Opt. 28, 3268-3270 (1989).

10. J. R. Buckland, J. M. Huntley, and S. R. E. Turner, "Unwrapping noisy phase maps by use of a minimum-cost-matching algorithm," Appl. Opt. 34, 5100-5108 (1995).

11. R. Cusack, J. M. Huntley, and H. T. Goldrein, "Improved noise-immune phase-unwrapping algorithm," Appl. Opt. 34, 781-789 (1995). 
12. M. Costantini, "A novel phase unwrapping method based on network programming," IEEE Trans. Geosci. Remote Sensing 36, 813-821 (1998).

13. B. Gutmann and H. Weber, "Phase unwrapping with the branch-cut method: clustering of discontinuity sources and reverse simulated annealing," Appl. Opt. 38, 5577-5593 (1999).

14. S. A. Karout, M. A. Gdeisat, D. R. Burton, and M. J. Lalor, "Two-dimensional phase unwrapping using a hybrid genetic algorithm," Appl. Opt. 46, 730-743 (2007).

15. D. L. Fried, "Least-square fitting a wave-front distortion estimate to an array of phase-difference measurements," J. Opt. Soc. Am. 67, 370-375 (1977).

16. R. H. Hudgin, "Wave-front reconstruction for compensated imaging," J. Opt. Soc. Am. 67, 375-378 (1977).

17. B. R. Hunt, "Matrix formulation of the reconstruction of phase values from phase differences," J. Opt. Soc. Am. 69, 393-399 (1979).

18. D. C. Ghiglia and L. A. Romero, "Direct phase estimation from phase differences using fast elliptic partial differential equation solvers," Opt. Lett. 14, 1107-1109 (1989).

19. H. Takajo and T. Takahashi, "Least-squares phase estimation from the phase difference," J. Opt. Soc. Am. A 5, 416-425 (1988).

20. H. Takajo and T. Takahashi, "Noniterative method for obtaining the exact solution for the normal equation in least-squares phase estimation from the phase difference," J. Opt. Soc. Am. A 5, 1818-1827 (1988).

21. D. C. Ghiglia and L. A. Romero, "Robust two-dimensional weighted and unweighted phase unwrapping that uses fast transforms and iterative methods," J. Opt. Soc. Am. A 11, 107-117 (1994).

22. D. J. Bone, H.-A. Bachor, and R. J. Sandeman, "Fringe-pattern analysis using a 2-D Fourier transform,” Appl. Opt. 25, 1653-1660 (1986).

23. R. Yamaki and A. Hirose, "Singularity-spreading phase unwrapping," IEEE Trans. Geosci. Remote Sensing 45, 3240-3251 (2007).

24. D. J. Bone, "Fourier fringe analysis: the two-dimensional phase unwrapping problem," Appl. Opt. 30, 3627-3632 (1991).

25. P. M. Morse and H. Feshbach, Methods of Theoretical Physics (McGraw-Hill, 1953), pp. 52-54.

26. S. Tomioka, S. Nisiyama, and T. Enoto, "Nonlinear least square regression by adaptive domain method with multiple genetic algorithms," IEEE Trans. Evol. Comput. 11, 1-16 (2007).

27. J. A. Quiroga, A. González-Cano, and E. Bernabeu, "Stablemarriages algorithm for preprocessing phase maps with discontinuity sources," Appl. Opt. 34, 5029-5038 (1995). 